\title{
An Algorithm to Estimate the ST Segment Level in 24-Hour Ambulatory ECG Records
}

\author{
A Smrdel, F Jager \\ University of Ljubljana, Faculty of Computer and Information Science, Ljubljana, Slovenia
}

\begin{abstract}
We present an algorithm to estimate the ST segment level, and to construct the ST segment level function. The algorithm was developed and tested using the Long-Term ST Database (LTST DB). The algorithm determines the positions of the isoelectric level and the J point in average heart beats constructed from 16-second windows of normal and non-noisy heart beats. Then the samples of the ST segment level function are derived for each ECG lead. The aggregate average error between the amplitudes of the samples of the ST segment level functions, for 190 ECG leads of the LTST DB, constructed automatically and those constructed on the basis of manually set positions of the isoelectric level and the J point by the human expert annotators of the database was $0.69 \mu \mathrm{V}$ (std. $8.89 \mu \mathrm{V})$.
\end{abstract}

\section{Introduction}

Ambulatory electrocardiogram (AECG) data typically shows wide and significant $(>50 \mu \mathrm{V})$ transient changes in amplitude of ST segment level. These changes appear due to slow drifts of ST segment level, changes of the electrical axis of the heart, changes in the ventricular conduction, heart-rate related changes, and ischemia. For reliable automatic detection of ischemic and other non-ischemic ST segment changes, which complicate detection of true ischemia, an accurate construction of the ST segment level function, representing a time trend of the ST segment level throughout the record, is an important step.

In this paper we present an algorithm for estimation of the ST segment level and for the construction of the ST segment level function. The algorithm was developed and tested using all 24-hour AECG records of the Long-Term ST Database (LTST DB) [1].

\section{Methods}

The LTST DB contains 86 2- and 3-channel AECG records of 80 patients, sampled at 250 samples per second ( $\Delta T=4 \mathrm{~ms}$ ) per channel, which were collected during regular clinical practice. The records of the LTST DB underwent a considerable automatic preprocessing phase during its development [1] which included: ARISTOTLE's analysis [2] yielding stable QRS complex fiducial points, noise removal, derivation of the instantaneous heart rate, automatic search for the positions of the isoelectric level, measurement of the ST segment level, derivation of the Karhunen-Loève transform based QRS complex and ST segment morphology feature vectors, removal of abnormal heart beats and their neighbors, and removal of noisy heart beats. After that the human expert annotators of the LTST DB manually determined the positions of the isoelectric level and the J point, which were then used to derive the ST segment level functions and the ST segment deviation functions. The ST segment deviation functions were obtained as the ST segment level functions from which the time-varying ST segment reference level functions (annotated manually) were subtracted. In the ST segment deviation functions the transient ischemic and transient nonischemic heart-rate related ST segment episodes were then manually annotated.

The input to the developed algorithm were raw ECG data of the records and the ARISTOTLE's fiducial points of normal and non-noisy hear beats (stored in *.dmy files of the LTST DB), which passed the LTST DB preprocessing phase. All these data are available to the users of the LTST DB. Initially the algorithm constructs average heart beat for each individual normal and non-noisy heart beat using normal and non-noisy heart beats in the 16-second neighborhood of the current heart beat. In these average heart beats the algorithm searches for the positions of the isoelectric reference points (IRPs) and the J points.

For the position of the IRP, $I(i, j)$, where $i$ denotes the lead number and $j$ denotes the heart beat number, a procedure initially searches from the ARISTOTLE's fiducial point, $F P(j)$, backwards in each ECG lead for up to 60 $\mathrm{ms}$ for a signal sample where the amplitude difference of two consecutive samples equals zero or changes sign. This sample may be the end of the P-Q interval, the R or the Q peak. The procedure then searches from this sample backwards to the point $F P(j)-108 \mathrm{~ms}$ for the "flattest" 20 $\mathrm{ms}$ interval of the waveform. The flattest $20 \mathrm{~ms}$ interval is defined as that interval, which yields minimum sum of 
the absolute deviations of signal samples taken from the intervals own mean.

Usual QRS complex width is about $80 \mathrm{~ms}$ but in the AECG records we often find heart beats with wider QRS complexes. On the other hand, the position of the ARISTOTLE's fiducial point is placed in the center of gravity of QRS complex what does not necessarily mean that it is placed close to the R peak, given lead, and distance to the IRP may be "far". Therefore, the procedure searches for the position of the IRP in two regimes [3]. For those records with wider QRS complexes, the procedure uses wider interval following: if the signal sample at which amplitude difference of two consecutive samples equals zero or changes sign is at least $48 \mathrm{~ms}$ from the $F P(j)$ for at least 40 out of the first 50 heart beats of the record in at least one ECG lead, then the interval for searching of the flattest interval is extended for all leads to $F P(j)-148$ $\mathrm{ms}$. The flattest interval found is then considered the NN interval containing the isoelectric level. The middle sample of the flattest interval defines the position of the IRP, $I(i, j)$. Next, the procedure calculates the average distance of the positions of the IRPs from the corresponding fiducial points for the last 16 heart beats, $\overline{D_{I}}(i, j)$, and compares it to the distance of the position of the current IRP, $D_{I}(i, j)$. If the $\overline{D_{I}}(i, j)$ and $D_{I}(i, j)$ differ for more than $8 \mathrm{~ms}$, the procedure searches again for the flattest $20 \mathrm{~ms}$ interval of the waveform in the $8 \mathrm{~ms}$ interval, this time from the $F P(j)-\overline{D_{I}}(i, j)$, in either direction, towards the $F P(j)-D_{I}(i, j)$. The middle sample of this $20 \mathrm{~ms}$ interval then defines the position of the IRP, $I(i, j)$, otherwise $I(i, j)$ remains as previously determined. By this, we prevent too fast changes of the $\overline{D_{I}}(i, j)$ which may occur due to erroneously determined positions of the IRP. Thus we ensure the robustness, and despite that, the procedure can still track slow changes of the position of the IRP [3].

Then the procedure determines the final position of the IRP for the $j$-th heart beat by comparing the positions of the IRPs found for all leads. If the positions of the IRP from at least two different leads differ for more than $8 \mathrm{~ms}$, the procedure sums the absolute deviations (from the $20 \mathrm{~ms}$ intervals) over all leads at each single lead position of the IRP. That position of the IRP of a lead, for which this sum over all leads is minimal is then taken as the final unique position of the IRP for all leads, $I(j)$, otherwise, the positions of the IRPs, $I(i, j)$, remain as previously determined in each ECG lead.

Next, another procedure searches for the position of the J point, $J(i, j)$. The procedure searches forward in each ECG lead from the $F P(j)$ for up to $32 \mathrm{~ms}$ for a signal sample, where the amplitude difference of two consecutive samples equals zero or changes sign. This may be the $\mathrm{R}$ or the $\mathrm{S}$ peak. From this point, or from the $F P(j)$ if such a sample was not found, the procedure searches forward for up to $68 \mathrm{~ms}$ for the interval of the waveform which "starts to flatten", i.e. the interval of the waveform, where the signal slope is "small enough". For this purpose, the procedure calculates, for each signal sample, the absolute amplitude difference between the mean of three preceding and three trailing samples. The first of the three consecutive signal samples for which this absolute difference is less than $15 \mu \mathrm{V}$ is considered to be the position of the $\mathrm{J}$ point, $J(i, j)$. The slope criterion was adopted from [4]. By this, we wanted to avoid erroneously determined positions of the $\mathrm{J}$ points in the leads [3]. If such a sample was not found, the procedure sets the position of the $\mathrm{J}$ point simply $40 \mathrm{~ms}$ after the $F P(j)$.

The unique position of the $\mathrm{J}$ point for the $j$-th heart beat, $J(j)$, is then taken as that position of the $\mathbf{J}$ point from the leads, which is the furthest from the $F P(j)$. The procedure next calculates the average distance of the positions of the $\mathrm{J}$ points from the corresponding fiducial points for the last 16 heart beats, $\overline{D_{J}}(j)$, and compares it to the distance of the position of the current $\mathbf{J}$ point, $D_{J}(j)$. If the $\overline{D_{J}}(j)$ and $D_{J}(j)$ differ for more than $8 \mathrm{~ms}$, then the final position of the $\mathbf{J}$ point is moved for $8 \mathrm{~ms}$, in either direction, towards the $F P(j)+\overline{D_{J}}(j)$, otherwise $J(j)$ remains as previously determined.

Using the positions of the IRP and the $\mathrm{J}$ point in the average heart beats, the algorithm then constructs the ST segment level function, $s(i, j)$, as:

$$
s(i, j)=a(i, j)-z(i, j),
$$

where $a(i, j)$ is the signal amplitude at the point of measurement of the ST segment level, $S(j)$, and $z(i, j)$ is the signal amplitude at the position of the IRP, $I(i, j)$. Both, the $a(i, j)$ and $z(i, j)$, are actually determined as means of amplitudes of signal samples surrounding $S(j)$ and $I(i, j)$ in the $20 \mathrm{~ms}$ interval. The $S(j)$ is determined according to the position of the $\mathrm{J}$ point, $J(j)$, and the heart rate, $H R(j)$, following [1]:

$$
S(j)=\left\{\begin{array}{l}
J(j)+80 \mathrm{~ms}: \text { if } \quad H R(j)<100 \mathrm{bpm} \\
J(j)+72 \mathrm{~ms}: \text { if } 100 \mathrm{bpm} \leq H R(j)<110 \mathrm{bpm} \\
J(j)+64 \mathrm{~ms}: \text { if } 110 \mathrm{bpm} \leq H R(j)<120 \mathrm{bpm} \\
J(j)+60 \mathrm{~ms} \text { : if } 120 \mathrm{bpm} \leq H R(j) .
\end{array}\right.
$$

\section{Results}

The algorithm was tested using all 86 records of the LTST DB. The errors between the automatically determined positions of the IRP and those manually determined by the expert annotators of the LTST DB are shown in Table 1. (The positions of the IRP and the $J$ point for each normal and non-noisy heart beat, as determined manually by the expert annotators of the LTST DB, are available to the users of the database and are stored in the *.16a files 

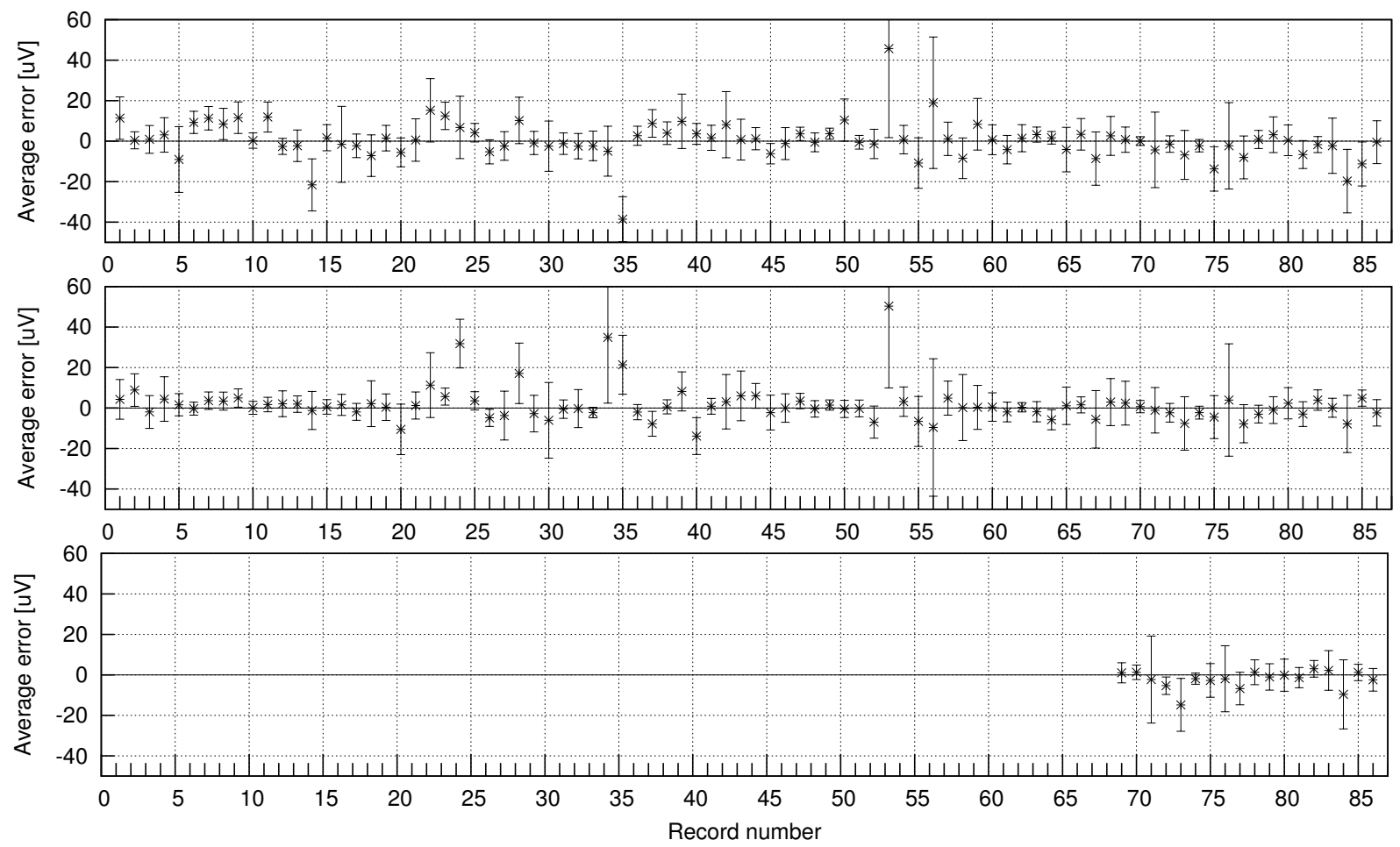

Figure 1. Results of the comparison of the amplitudes of the samples of the ST segment level functions constructed on the basis of automatically and manually determined positions of the IRP and the J point for the leads 0 (top), 1 (middle), and 2 (bottom) of the LTST DB. Average errors with standard deviations are shown.

Table 1. The aggregate average errors and standard deviations of automatically and manually determined positions of the IRP, of the J point, and of the amplitudes of the samples of the ST segment level functions obtained using automatically and manually determined positions of the IRP and $\mathbf{J}$ point, for leads 0,1 , and 2 , and for all leads of the LTST DB.

\begin{tabular}{lrrr}
\hline & IRP & J point & ST segment level \\
\hline Lead 0 & $-2.80 \mathrm{~ms}$ & $-2.04 \mathrm{~ms}$ & $0.38 \mu \mathrm{V}$ \\
St. Dev. & $7.56 \mathrm{~ms}$ & $5.04 \mathrm{~ms}$ & $9.17 \mu \mathrm{V}$ \\
\hline Lead 1 & $-3.60 \mathrm{~ms}$ & $-3.84 \mathrm{~ms}$ & $1.60 \mu \mathrm{V}$ \\
St. Dev. & $7.76 \mathrm{~ms}$ & $5.16 \mathrm{~ms}$ & $8.73 \mu \mathrm{V}$ \\
\hline Lead 2 & $-2.72 \mathrm{~ms}$ & $-5.84 \mathrm{~ms}$ & $-2.23 \mu \mathrm{V}$ \\
St. Dev. & $6.68 \mathrm{~ms}$ & $5.92 \mathrm{~ms}$ & $8.27 \mu \mathrm{V}$ \\
\hline All Leads & $-3.16 \mathrm{~ms}$ & $-3.20 \mathrm{~ms}$ & $0.69 \mu \mathrm{V}$ \\
St. Dev. & $7.56 \mathrm{~ms}$ & $5.20 \mathrm{~ms}$ & $8.89 \mu \mathrm{V}$ \\
\hline
\end{tabular}

of the database.) The aggregate average error for all 190 leads of the LTST DB was $-3.16 \mathrm{~ms}$, with the standard deviation of $7.56 \mathrm{~ms}$. The aggregate average error for automatically and manually determined positions of the J point for all 190 leads of the database was $-3.20 \mathrm{~ms}$, with standard deviation of $5.20 \mathrm{~ms}$ (see Table 1). Errors are in both cases relatively small considering the fact, that the time step between the signal samples is only $4 \mathrm{~ms}$. The aggregate average error between the amplitudes of the samples of the ST segment level functions derived using automati- cally and manually determined positions of the IRP and the $\mathrm{J}$ point for all 190 leads of the database was $0.69 \mu \mathrm{V}$, with the standard deviation of $8.89 \mu \mathrm{V}$ (see Table 1). The error is small, indicating high performance of the developed algorithm. But higher standard deviation indicates that the ST segment level measurements obtained using automatically determined positions oscillate around measurements obtained using manually determined positions.

Figure 1 shows results of the comparison of the amplitudes of the samples of the ST segment level functions derived using automatically and manually determined positions of the IRP and the $\mathrm{J}$ point for all 86 records of the LTST DB. The average error is relatively small for the most leads (under $10 \mu \mathrm{V}$ ), with only 5 leads out of 190 , where the average error exceeds $25 \mu \mathrm{V}$. These records are: the record 35 (s20321) and 53 (s20501) in the lead 0, and the record 24 (s20241), 34 (s20311), and 53 (s20501) in the lead 1.

Figure 2 shows the ST segment level functions for the leads of the record 1 (s20011) of the LTST DB. The visual inspection and comparison of the ST segment level functions derived using automatically and manually determined positions of the IRP and the J point shows that they resemble each other quite well. In the ST segment level functions derived using automatically determined positions there are no apparent significant artifacts, what enables reliable further analysis and detection of transient ST 


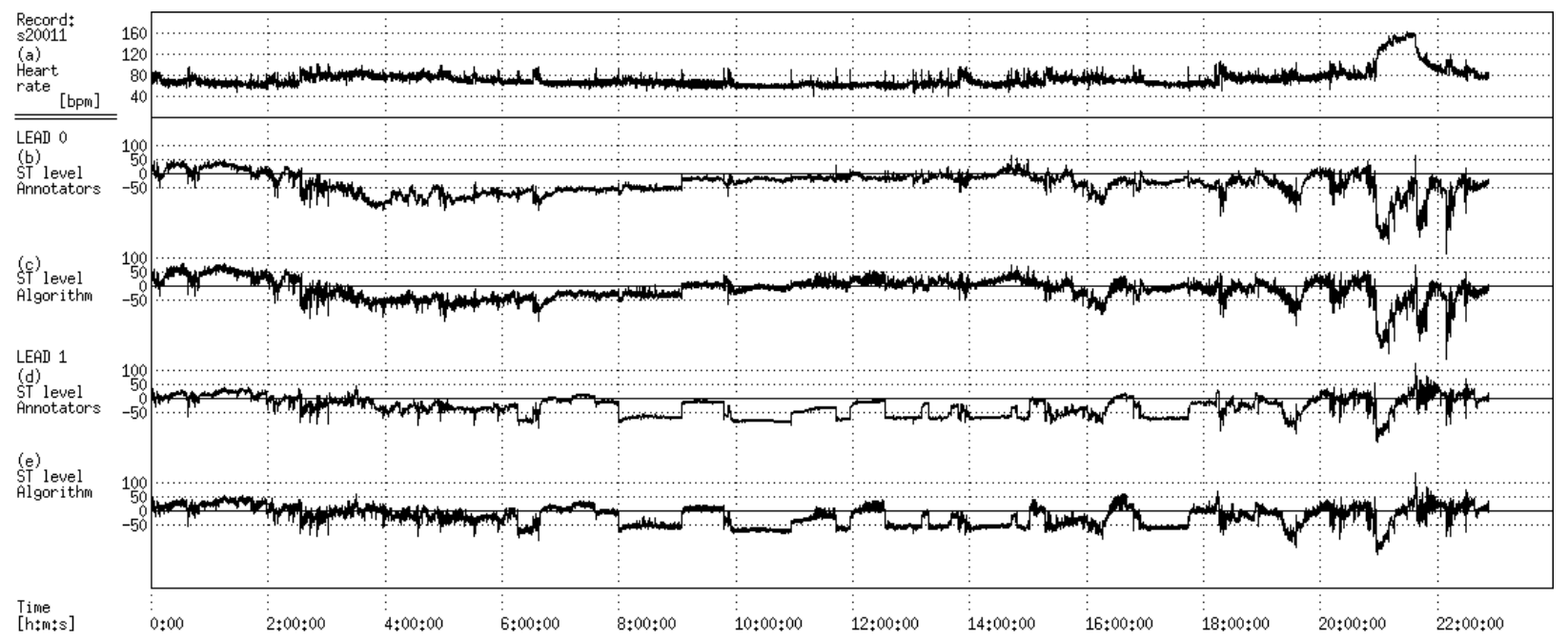

Figure 2. The ST segment level functions of the record s20011 (refer also to Figure 1, record number 1) of the LTST DB. Legend: (a) heart rate [bpm]; (b) and (d) the ST segment level functions for the lead 0 and 1, respectively, constructed using manually determined positions (see text) $[\mu \mathrm{V}]$; (c) and (e) the ST segment level functions for the lead 0 and 1 , respectively, constructed using automatically determined positions $[\mu \mathrm{V}]$. The average errors (the lead 0 and 1, respectively, standard deviations are bracketed) for the positions of the IRP were: $-11.16 \mathrm{~ms}(7.52 \mathrm{~ms})$ and $-7.40 \mathrm{~ms}$ (7.56 ms); for the positions of the J point were: $1.56 \mathrm{~ms}(4.4 \mathrm{~ms})$ and $0.24 \mathrm{~ms}(3.72 \mathrm{~ms})$; and for the amplitudes of the samples of the ST segment level functions were: $11.38 \mu \mathrm{V}(10.49 \mu \mathrm{V})$ and $4.30 \mu \mathrm{V}(9.79 \mu \mathrm{V})$.

segment episodes. Only a few non-significant discrepancies in the ST segment level functions may be seen in this record at approximately 4 hours.

\section{Discussion and conclusions}

The annotators of the LTST DB set unique position of the IRP for each heart beat, over all leads, in some records (these are: s20231, s20251, s20272, s20391, s20411, and records from s20581 to s30801), while in rest of records they set the positions for each lead separately. They also set the unique positions of the $\mathrm{J}$ point for each heart beat, over all leads, in the records from s20581 to s30801, while in the rest of records they set the positions separately. The developed algorithm sets the position of the IRP in each heart beat for each lead separately, except in the case where the "flat" intervals in different leads are wide apart. The positions of the IRP that are found wide apart may occur due to the erroneous or noisy signal waveforms so the algorithm sets robustly the unique IRP for all leads in such cases. On the other hand, the developed algorithm always sets the unique position of the $\mathrm{J}$ point.

Clinically significant ST segment changes are those with the amplitude change greater than $50 \mu \mathrm{V}$. The aggregate average error of the amplitudes of the samples of the ST segment level functions obtained using automatically and manually determined positions of the IRP and the $\mathrm{J}$ point is relatively small, $0.69 \mu \mathrm{V}$, with the standard deviation under $10 \mu \mathrm{V}$, what is much less than the significant ST segment change.
In conclusion, we developed a simple, accurate and efficient algorithm for automatic search for the positions of the IRP and the J point, estimation of the ST segment level and construction of the ST segment level functions, and furthermore for the detection of transient ST segment change episodes.

\section{References}

[1] Jager F, Taddei A, Moody GB, Emdin M, Antolič G, Dorn R, Smrdel A, Marchesi C, Mark RG. Long-term ST database: a reference for the development and evaluation of automated ischaemia detectors and for the study of the dynamics of myocardial ischaemia. Med Biol Eng Comp 2003;41:172-182.

[2] Moody GB, Mark RG. Development and evaluation of a 2lead ECG analysis program. In Computers in Cardiology 1982. Los Alamitos: IEEE Computer Society Press, 1982; $39-44$.

[3] Smrdel A. Robust automated detection of transient ST segment episodes in 24-hour electrocardiograms. Ph.D. thesis, University of Ljubljana, Faculty of Computer and Information Science, 2004.

[4] Daskalov IK, Dotsinsky IA, Christov II. Development in ECG acquisition, preprocessing, parameter measurement, and recording. IEEE Eng Med Biol 1998;50-58.

Address for correspondence:

Aleš Smrdel

University of Ljubljana, Fac. of Computer and Information Sci.

Tržaška 25, 1000 Ljubljana, Slovenia

ales.smrdel@fri.uni-lj.si 